\title{
Magnetite-based nanomedicine for cancer diagnosis and therapy using loco-regional hyperthermia combined with chemotherapy
}

\author{
Shu-Jyuan Yang1, Shu-Yi Tseng', Chung-Hao Wang², Tai-Horng Young1, and Ming-Jium Shieh ${ }^{1}$ \\ ${ }^{1}$ Institute of Biomedical Engineering, College of Medicine and College of Engineering, National Taiwan University \\ No. 1, Section 1, Jen-Ai Road, Taipei 100, Taiwan \\ image0120@gmail.com; angel01040218@gmail.com; thyoung@ntu.edu.tw; soloman@ntu.edu.tw \\ ${ }^{2}$ Gene'e Tech Co. Ltd. \\ 2F., No.661, Bannan Rd., Zhonghe Dist., New Taipei City 235, Taiwan \\ wangenee@gmail.com
}

\section{Extended Abstract}

Colorectal cancer is now one of the major diseases in the world. With westernized diet in Taiwan, the incidence of colorectal cancer increases. For cancer stem cell therapy, CD133 (prominin-1) is a theoretical cancer stem cell (CSC) marker for colorectal cancer and is a proposed therapeutic target. Cells with CD133 overexpression have demonstrated enhanced tumor-initiating ability and tumor relapse probability [1-6]. To resolve the problem of chemotherapy failure, we will develop a magnetite-based nanomedicine using loco-regional hyperthermia combined with chemotherapy. The targeting carrier has a magnetite nanoparticle (superparamagnetic iron oxide nanoparticles, SPIO) core and a layer-by-layer polyelectrolyte molecule shell that carries irinotecan (CPT-11) and anti-human prominin-1 (PROM1/CD133) monoclonal antibody for cancer stem cell-specific targeting. Besides as a contrast agent for MRI, this nanomedicine plays as an important role to relay the externally delivered radiofrequency energy for tumor hyperthermia [7,8]. Locoregional heat can trigger a drug release from the carrier as it directly damages tumor cells and cancer stem cells. .Finally, the use of this nanomedicine can improve the half-life of chemotherapy drugs in the blood and reduce the side effect, and is significantly more efficacious than hyperthermia or chemotherapy alone for colorectal cancer therapy.

\section{References}

[1] Y. Wu and P. Y. Wu, "CD133 as a marker for cancer stem cells: progresses and concerns," Stem Cells Dev., vol. 18, pp. 1127-1134, 2009.

[2] C. Zhang, C. Zhou, X. J. Wu, et al., "Human CD133-positive hematopoietic progenitor cells initiate growth and metastasis of colorectal cancer cells," Carcinogenesis., vol. 35, pp. 2771-2777, 2014.

[3] T. Baba, P. A. Convery, N. Matsumura, et al., "Epigenetic regulation of CD133 and tumorigenicity of CD133+ ovarian cancer cells," Oncogene., vol. 28, pp. 209-218, 2009.

[4] J. M. Yi, H. C. Tsai, S. C. Glöckner, et al., "Abnormal DNA methylation of CD133 in colorectal and glioblastoma tumors," Cancer Res., vol. 68, pp. 8094-8103, 2008.

[5] K. Hibi, M. Sakata, Y. H. Kitamura, et al., "Demethylation of the CD133 gene is frequently detected in early gastric carcinoma," Anticancer Res., vol. 30, pp. 1201-1203, 2010.

[6] D. Corbeil, A. M. Marzesco, M. Wilsch-Brauninger, et al., "The intriguing links between prominin-1 (CD133), cholesterol-based membrane microdomains, remodeling of apical plasma membrane protrusions, extracellular membrane particles, and (neuro)epithelial cell differentiation," FEBS Lett., vol. 584, pp. 1659-1664, 2010.

[7] G. Tan, C. Chia, M. Kumar, S. P. Choo, et al. "201 consecutive cytoreductive surgery (CRS) and hyperthermic intraperitoneal chemotherapy (HIPEC) procedures in a single Asian tertiary centre," Int. J. Hyperthermia, vol. 14, pp. 1-7, 2016.

[8] N. R. Datta, S. Krishnan, D. E. Speiser, et al., "Magnetic nanoparticle-induced hyperthermia with appropriate payloads: Paul Ehrlich's "magic (nano)bullet" for cancer theranostics?" Cancer Treat Rev., vol. 50, pp. 217-227, 2016. 